\title{
Real-Analytic Negligibility of Points and Subspaces in Banach Spaces, with Applications
}

\author{
D. Azagra and T. Dobrowolski
}

\begin{abstract}
We prove that every infinite-dimensional Banach space $X$ having a (not necessarily equivalent) real-analytic norm is real-analytic diffeomorphic to $X \backslash\{0\}$. More generally, if $X$ is an infinitedimensional Banach space and $F$ is a closed subspace of $X$ such that there is a real-analytic seminorm on $X$ whose set of zeros is $F$, and $X / F$ is infinite-dimensional, then $X$ and $X \backslash F$ are real-analytic diffeomorphic. As an application we show the existence of real-analytic free actions of the circle and the $n$-torus on certain Banach spaces.
\end{abstract}

In 1951 Victor Klee proved that, if $X$ is either a non-reflexive Banach space or an infinite-dimensional $L^{p}$ space and $K$ is a compact subset of $X$ then $X \backslash K$ and $X$ are homeomorphic. He also showed that every infinite-dimensional Hilbert space is homeomorphic to its unit sphere, and he gave a complete topological classification of the convex bodies of a Hilbert space. These results were later extended to the class of all infinite-dimensional Banach spaces by Bessaga and Klee (cf. [6], [8], [9], [10]). If a subset $A$ of $X$ has the property that $X$ and $X \backslash A$ are homeomorphic, we say that $A$ is negligible. It is natural to ask whether this type of results can be sharpened so as to get diffeomorphisms instead of merely homeomorphisms.

In 1966, C. Bessaga [5] proved that if $X$ is an infinite-dimensional Hilbert space then $X$ is $C^{\infty}$ diffeomorphic to both $X \backslash\{0\}$ and its unit sphere. Some twelve years later, the second-named author [16] developed the so-called non-complete norm technique of Bessaga's in the smooth case and showed that if $X$ has a non-complete $C^{p}$ smooth norm then $X$ and $X \backslash K$ are $C^{p}$ diffeomorphic for any compact set $K \subset X$. Unfortunately, it is not known whether every infinite-dimensional space with an equivalent $C^{p}$ smooth norm must have a non-complete $C^{p}$ smooth norm too, so that this result does not allow us to conclude that the same holds true for all infinitedimensional Banach spaces with smooth norms.

Without proving the existence of smooth non-complete norms, the first-named author recently showed [2] that every infinite-dimensional Banach space with a (not necessarily equivalent) $C^{p}$ smooth norm $\varrho$ is $C^{p}$ diffeomorphic to $X \backslash\{0\}$ and, furthermore, that every hyperplane in $X$ is $C^{p}$ diffeomorphic to the sphere $\{x \in X \mid$ $\varrho(x)=1\}$. Then the present authors strengthened the asymmetric norm technique of deleting points introduced in [2] so as to obtain very general results concerning smooth negligibility of compact sets and subspaces [3]. These results allow to enlarge the class of spaces in which some striking applications of negligibility theory are valid (see [3], [4], [7], [19], [20], [21], [25]). 
The real-analytic negligibility of compact sets and subspaces in separable Banach spaces was studied by the second-named author [16]. He proved that if $X$ is a separable infinite-dimensional Banach space and $A$ is either a compact subset of $X$ or a closed subspace with $\operatorname{dim}(X / A)=\infty$, then $X$ and $X \backslash A$ are real-analytic diffeomorphic. It is natural to ask whether such results will remain valid in the non-separable case. The answer is negative in general: if $\Gamma$ is an uncountable set then it can be shown that $c_{0}(\Gamma)$ is not real-analytic diffeomorphic to $c_{0}(\Gamma) \backslash\{0\}$ (see Proposition 4.7 in [16]). However, by analogy with what happens in the smooth case [3], it seems natural to conjecture that, if $F$ is a closed subspace which is the set of zeros of a realanalytic seminorm on a space $X$, and $\operatorname{dim}(X / F)=\infty$, then $X$ and $X \backslash F$ must be real-analytic diffeomorphic.

In this note we show this conjecture to be true, giving explicit formulae for realanalytic diffeomorphisms between an infinite-dimensional Banach space $X$ and the space minus an infinite-codimensional subspace $F$, provided the space $X$ has a realanalytic seminorm whose set of zeros is $F$. As a result, singletons are real-analytic negligible in every infinite-dimensional Banach space having a real-analytic norm. It should be noted that the class of Banach spaces having (not necessarily equivalent) real-analytic norms is large. For instance, it is easy to show that every Banach space which is linearly injectable into some $\ell_{p}(\Gamma)(1<p<\infty)$ has a (not necessarily equivalent) real-analytic norm. Taking into account that every superreflexive Banach space is linearly injectable into some $\ell_{p}(\Gamma)$ with $1<p<\infty$ (see [24, proof of Lemma 2, p. 133]), and the same is true for all separable spaces, it follows that all superreflexive spaces and all separable spaces have such norms.

At the end of the paper we give some applications of these results concerning realanalytic free actions of some Lie groups (such as the unit circle and the $n$-torus) on certain Banach spaces (namely, those which have separable complemented subspaces which are isomorphic to their cartesian squares). As a corollary it is deduced that such spaces have real-analytic self-diffeomorphisms of arbitrary period with no fixed points.

Let us formally state the main result of this paper.

Theorem 1 Let $(X,\|\cdot\|)$ be an infinite-dimensional Banach space with a real-analytic seminorm $\varrho$ whose set of zeros is a subspace F such that the quotient space $X / F$ is infinitedimensional. Then there exists a real-analytic diffeomorphism between $X$ and $X \backslash F$.

In particular, we have:

Corollary 2 Let $X$ be an infinite-dimensional Banach space with a (not necessarily equivalent) real-analytic norm. Then there exists a real-analytic diffeomorphism between $X$ and $X \backslash\{0\}$.

The proof of Theorem 1 is rather technical. We will make use of some auxiliary results which we state and prove next. The gist of the proof is as follows. We will construct a certain real-analytic path $p$ on $X$ and a certain function $\omega: X \longrightarrow[0, \infty)$ such that $\omega^{-1}(0)=F$ and $\omega$ is real-analytic on $X \backslash F$ (the function $\omega$ will be different depending upon whether the space is reflexive or not), and we will show that the 
formula $\psi(x)=x+p(\omega(x)), x \in X \backslash F$, establishes a real-analytic diffeomorphism between $X \backslash F$ and $X$. In order to show that $\psi$ is a bijection we make use of Lemma 3. Lemma 4 shows that our path $p$ is real-analytic, while Lemma 5 is a technical fact which will help us construct the function $\omega$. The proofs are not detailed and the reader is supposed to have some knowledge of the techniques introduced in [2], [3] in order to fill in the gaps in the arguments (he might also want to consult [1], where very detailed proofs are given).

Lemma 3 Let $F:(0, \infty) \longrightarrow[0, \infty)$ be a continuous function such that, for every $\beta \geq \alpha>0, F(\beta)-F(\alpha) \leq \frac{1}{2}(\beta-\alpha)$ and $\lim \sup _{t \rightarrow 0^{+}} F(t)>0$. Then there exists $a$ unique $\alpha>0$ such that $F(\alpha)=\alpha$.

Lemma 4 Let $(X,\|\cdot\|)$ be a Banach space, and let $\left(y_{k}\right)$ be a sequence of vectors such that $\left\|y_{k}\right\| \leq 1$ for all $k$. Consider the function $G: \mathbb{R} \longrightarrow \mathbb{R}, G(s)=\frac{1}{1+s^{2}}$, and define the path $p:(0, \infty) \longrightarrow X$ by

$$
p(t)=\sum_{k=1}^{\infty} G\left(2^{k-1} t\right) y_{k} .
$$

Then $p$ is a real-analytic function from $(0, \infty)$ to $X$.

Proof Let $Y$ be the complexification of the space $X$, and let $\Omega=\{z \in \mathbb{C}: \operatorname{Re} z>$ $2|\operatorname{Im} z|\}$, which is an open subset of the complex plane containing the interval $(0, \infty)$ of the real line. Since the zeros of the complex function $z \longrightarrow \frac{1}{1+4^{k-1} z^{2}}$ are outside $\Omega$ for each $k \in \mathbb{N}$, every function

$$
g_{k}(z)=\frac{y_{k}}{1+4^{k-1} z^{2}}=G\left(2^{k-1} z\right)
$$

is holomorphic in $\Omega$. It is not difficult to see that the series $\sum_{k=1}^{\infty} g_{k}$ converges uniformly on the compact subsets of $\Omega$ to a holomorphic function $p: \Omega \longrightarrow Y$. In particular, the restriction of $p$ to the interval $(0, \infty)$ of the real line is real-analytic.

Recall that, for a real linear space $X$, a function $\varrho: X \longrightarrow[0, \infty)$ is said to be a seminorm in $X$ provided $\varrho$ satisfies the following properties:

(i) $\varrho(x+y) \leq \varrho(x)+\varrho(y)$ for every $x, y \in X$; and

(ii) $\varrho(\lambda x)=|\lambda| \varrho(x)$ for every $x \in X$ and every real number $\lambda$; in particular, $\varrho(0)=$ 0 .

In the proof of Theorem 1 we will handle other functionals belonging to a quite larger class, namely those functionals $\omega: X \longrightarrow[0, \infty)$ satisfying

(1) $\omega(x+y) \leq \omega(x)+\omega(y)$ for every $x, y \in X$; and

(2) $\omega(\lambda x)=\lambda \omega(x)$ for every $x \in X$ and every $\lambda \geq 0$. 
Note that such a functional $\omega$ need not be a seminorm; in general $\omega(-x) \neq \omega(x)$. We will say that $\omega$ is an asymmetric seminorm. This does not mean that $\omega$ must always satisfy $\omega(-x) \neq \omega(x)$ for some $x$. It may be so, or it may not. Therefore, the class of "asymmetric seminorms" contains that of "seminorms". If, moreover, $\omega$ satisfies the property

(3) $\omega(x)=0$ if and only if $x=0$,

then we will call $\omega$ an asymmetric norm.

Next we state some of the properties of asymmetric seminorms that will be used in that proof. Let $\omega$ be an asymmetric seminorm in a linear space $X$, let $F=\omega^{-1}(0)$ be its set of zeros, and assume that $F$ is a linear subspace of $X$. Consider the canonical projection $\pi: X \longrightarrow X / F$. It is clear that $\omega$ also has the following property in this case: $\omega(x+z)=\omega(x)$ for every $z \in F, x \in X$. Hence $\omega$ induces a quotient functional $\bar{\omega}: X / F \longrightarrow[0, \infty)$ satisfying (1), (2) and (3) above, that is, an asymmetric norm, and such that $\omega=\bar{\omega} \circ \pi$. It is worth noting that the asymmetric norm $\bar{\omega}$ induced by a real-analytic asymmetric seminorm $\omega$ in the quotient space $X / \omega^{-1}(0)$ (when $\omega^{-1}(0)$ is a linear subspace) is real-analytic too. This fact, which is probably known and is not difficult to prove, will be used in the proof of Theorem 1 .

Lemma 5 Let $\omega: X \longrightarrow[0, \infty)$ be a functional satisfying (1) and (2) above and such that $F=\omega^{-1}(0)$ is a linear subspace of $X$. Suppose that $\omega$ is real-analytic (resp. $C^{n}$ smooth) on $X \backslash F$. Then, the induced quotient functional $\bar{\omega}: X / F \longrightarrow[0, \infty)$ is also real-analytic (resp. $C^{n}$ smooth) on $(X / F) \backslash\{\overline{0}\}$.

Now we are ready to give a proof of Theorem 1 . We will split the proof into two propositions, depending on whether or not our space is reflexive.

Proposition 6 Let $(X,\|\cdot\|)$ be an infinite-dimensional Banach space with a realanalytic seminorm $\varrho$ whose set of zeros is a subspace $F$ such that the normed space $(X / F, \bar{\varrho})$ is non-reflexive. Then there exists a real-analytic diffeomorphism between $X$ and $X \backslash F$.

Proof Let $\pi, \bar{\varrho}$ and $F$ be as in the definitions preceding the statement of Lemma 5. Since the normed space $(X / F, \varrho)$ is non-reflexive, according to James's theorem [23], there exists a linear functional $S: X / F \longrightarrow \mathbb{R}$ which is continuous from $(X / F, \bar{\varrho})$ onto $\mathbb{R}$ and such that $S$ does not attain the supremum $\sup \{S(\bar{z}) \mid \bar{z} \in X / F, \bar{\varrho}(\bar{z})=$ $1\}=1$. It should be noted that the norm $\varrho$ is continuous with respect to the usual quotient norm in $X / F$ (recall that $\varrho$ is real-analytic by virtue of Lemma 5 , and hence continuous in $X / F)$. Therefore the linear functional $S$ is also continuous from $X / F$ (with its usual quotient norm) onto $\mathbb{R}$.

Next, put $T=S \circ \pi \in X^{*}$, and define the functionals $\bar{\omega}: X / F \longrightarrow[0, \infty)$ and $\omega: X \longrightarrow[0, \infty)$ by

$$
\bar{\omega}(x)=\bar{\varrho}(\bar{x})-S(\bar{x}), \quad \text { and } \quad \omega(x)=\varrho(x)-T(x)=\bar{\omega}(\pi(x)) .
$$


It is quite clear that the functionals $\bar{\omega}$ and $\omega$ are real-analytic on the sets $(X / F) \backslash\{\overline{0}\}$ and $X \backslash F$ respectively. We can select vectors $\left(y_{k}\right)$ of $X$ such that $\varrho\left(y_{k}\right)=1$ and $\omega\left(y_{k}\right) \leq 1 / 4^{k}$ for every $k$. For $t>0$, write $G(t)=1 /\left(1+t^{2}\right)$ and consider the path

$$
p(t)=\sum_{k=1}^{\infty} G\left(2^{k-1} t\right) y_{k} .
$$

According to Lemma 4 , the path $p:(0, \infty) \longrightarrow X$ is real-analytic. Now, let us define $H: X \backslash F \longrightarrow X$ by

$$
H(x)=x+p(w(x)) .
$$

It is not difficult to check that for every $y \in X$ the function $F_{y}:(0, \infty) \longrightarrow[0, \infty)$ defined by $F_{y}(\alpha)=\omega(y-p(\alpha))$ satisfies the conditions of Lemma 3 and, therefore, has a unique fixed point $\alpha=\alpha(y)>0$. This means that the mapping $H$ is one-toone from $X \backslash F$ onto $X$, with

$$
H^{-1}(y)=y-p(\alpha(y)) .
$$

As the functions $\omega$ and $p$ are real-analytic, so is $H$. Finally, by using the real-analytic version of the implicit function theorem (see e.g. [15] and [28]) as in [3] we obtain that the mapping $y \rightarrow \alpha(y)$ is real-analytic and therefore $H: X \backslash F \longrightarrow X$ is a realanalytic diffeomorphism.

Proposition 7 Let $(X,\|\cdot\|)$ be an infinite-dimensional Banach space with a realanalytic seminorm $\varrho$ whose set of zeros is a subspace $F$ such that the normed space $(X / F, \bar{\varrho})$ is infinite-dimensional and reflexive. Then there exists a real-analytic diffeomorphism between $X$ and $X \backslash F$.

Proof Let us denote $Z=X / F$, and let $\pi: X \longrightarrow Z$ be the canonical projection. By Lemma 5 , the norm $\varrho: Z \longrightarrow \mathbb{R}$ induced by a real-analytic seminorm $\varrho$ satisfying $\varrho^{-1}(0)=F$ is also real-analytic on $Z$. In particular, the norm $\varrho$ is continuous with respect to the usual quotient norm of $Z=X / F$. On the other hand, the norm $\varrho$ is complete (indeed, the normed space $(X / F, \bar{\varrho})$ is reflexive and, hence, complete). Then, $\varrho$ is an equivalent real-analytic norm on $Z$. Consequently, the space $Z$ is $C^{\infty}$ smooth and, since it is reflexive, Theorem 4.1 in Chapter $\mathrm{V}$ of [13] gives us a $2 k$ homogeneous polynomial $h$ on $Z$ and constants $K, L>0$ such that $K \bar{\varrho}(z)^{2 k} \leq h(z) \leq$ $L \varrho(z)^{2 k}$ for every $z \in Z$; in particular, for such real-analytic $h: Z \rightarrow[0, \infty)$, we have $h^{-1}(0)=0$. According to [14, Theorem 3, p. 149], every reflexive (in general, every WCG) Banach space has a separable infinite-dimensional complemented subspace. Then we can write $Z=W \times V$, where $W$ is a separable infinite-dimensional subspace of $Z$. Since $W$ is separable, $W$ admits a non-complete norm $g$ such that $g^{2}$ is realanalytic on the whole of $W$ (see [16, Proposition 4.1]). For every $z=(u, v) \in Z=$ $W \times V$, let us define

$$
Q(z)=\sqrt{g(u)^{2}+h(v)} .
$$

It is clear that the function $Q: Z \rightarrow[0, \infty)$ is real-analytic on $Z \backslash\{0\}$ and satisfies $\left.Q\right|_{W}=g$ and $Q^{-1}(0)=0$. Since the norm $g$ is non-complete we can find a $\bar{\varrho}^{-}$ bounded sequence $\left(u_{k}\right)$ in $W$ such that $g\left(u_{k}\right) \leq \frac{1}{4^{k+1}}$ for each $k$, and a point $u_{0}$ in 
the completion of $(W, g)$, denoted by $(\hat{W}, \hat{g})$, such that $u_{0} \notin W$, and $\lim _{n} g\left(u_{0}-\right.$ $\left.\sum_{k=1}^{n} u_{k}\right)=0$. Let us choose a bounded sequence $\left(x_{k}\right)$ in $X$ such that $\pi\left(x_{k}\right)=\left(u_{k}, 0\right)$ for every $k$, put $G(t)=1 /\left(1+t^{2}\right)$, and define a path $q:(0, \infty) \longrightarrow X$ by

$$
q(t)=\sum_{k=1}^{\infty} G\left(2^{k-1} t\right) x_{k}
$$

for $t>0$. By Lemma 4, the path $q$ is real-analytic. Now define $H: X \backslash F \longrightarrow X$ by

$$
H(x)=x+q(Q(\pi(x)))
$$

for each $x \in X \backslash F$. As in the proof of the preceding proposition, it is not hard to see that $H: X \backslash F \longrightarrow X$ is a bijection, and both $H$ and $H^{-1}$ are real-analytic (see [1] for details).

By combining Propositions 6 and 7, we obtain Theorem 1.

V. L. Klee used his results on negligibility [25] to prove that for the Hilbert space $H$ and for each integer $n \geq 2$ there exists a periodic homeomorphism $f: H \longrightarrow H$ of pure period $n$ that has no fixed points. This was somewhat surprising because, for a finite-dimensional space $X$, P. A. Smith [26] had proved that every prime-periodic homeomorphism of $X$ must have a fixed point. By using Theorem 1, in many Banach spaces these results can now be improved so as to obtain real-analytic diffeomorphisms of arbitrary period $n$ having no fixed points. This holds for every Banach space having a complemented separable subspace which is isomorphic to its cartesian square. In fact these real-analytic version of Klee's results are obtained as corollaries to new results on free actions of the $n$-torus on Banach spaces.

Let us recall that a Lie group $G$ is said to act on a space $X$ if there exists a continuous map $\Phi: G \times X \rightarrow X$ such that $\Phi(e, x)=x$ and $\Phi(g h, x)=\Phi(g, \Phi(h, x))$ for all $g, h \in G$ and all $x \in X$. Here $e$ denotes the neutral element of the group $G$. If $X$ is a real-analytic manifold and $\Phi$ is real-analytic then we say that $G$ acts on $X$ in a real-analytic way. In such a case, for every $g \in G, x \mapsto \Phi(g, x)$ is a real-analytic self-diffeomorphism of $X$ (and $G$ can be identified with a subgroup of the group of diffeomorphisms of $X)$. If for every $g \neq e$ and $x \in X$ we have $\Phi(g, x) \neq x$, then the action is called free. Hereafter $T$ denotes the unit circle $\{s \in \mathbb{C}:|s|=1\}$, and $T^{n}$ stands for the $n$-torus $\left\{\left(s_{1}, \ldots, s_{n}\right) \in \mathbb{C}^{n}:\left|s_{j}\right|=1, j=1, \ldots, n\right\} ; T^{n}$ will be considered with its natural group structure.

Theorem 8 Let $X$ be a Banach space of the form $X=Y \times Z$, where $Z$ is a separable infinite-dimensional space which is isomorphic to its cartesian square. Then, for each $n \in \mathbb{N}$, there exists a real-analytic free action $\Phi$ of the $n$-torus $T^{n}$ on $X$.

Proof For the sake of simplicity we will write the proof only for the case $n=2$. The reader will immediately see that the same argument, with obvious modifications, holds in the general case. Since the space $Z$ is separable we can take a separating 
sequence of continuous functionals $\left(z_{n}^{*}\right) \subset Z^{*}$ such that $\left\|z_{n}^{*}\right\|=1$ for every $n$. Define $\omega: Z \times Z \longrightarrow[0, \infty)$ by

$$
\omega(u, v)=\left(\sum_{n=1}^{\infty} \frac{1}{2^{n}}\left(\left|z_{n}^{*}(u)\right|^{2}+\left|z_{n}^{*}(v)\right|^{2}\right)\right)^{1 / 2},
$$

where $(u, v) \in Z \times Z$. It is clear that $\omega$ is a prehilbertian norm in $Z \times Z$ which is compatible with the natural complex structure that the isomorphy of $Z$ and $Z \times$ $Z$ induces on $Z$ (as the formula $\omega(u+i v)=\omega(u, v)$ defines a norm on $Z$ when considered as a complex space). Choose a linear isomorphism $L: Z \longrightarrow Z \times Z$, define a prehilbertian norm $\varrho: Z \longrightarrow[0, \infty)$ by $\varrho(z)=\omega(L(z))$, and consider the $\varrho$-sphere of $Z, S=\{z \in Z \mid \varrho(z)=1\}$. In this setting, according to [17], there exists a real-analytic diffeomorphism from $Z$ onto $S \times \mathbb{R}$. Using once again the fact that $Z$ is isomorphic to $Z \times Z$, it is clear that there exists a real-analytic diffeomorphism between $Z$ and $\mathbb{R}^{2} \times S \times S$. By means of the isomorphism $L: Z \longrightarrow Z \times Z$ we may identify the sphere $S$ with the sphere $\hat{S}=\{u+i v \mid \omega(u, v)=1\}$ of the complex space $Z$. As noticed above, the norm $\omega$ is complex-symmetric, that is, for every complex $s$ with $|s|=1$, and for every $z=u+i v \in \hat{S}$, the product $s z$ belongs to $\hat{S}$. We have the following natural real-analytic free action of $T^{2}$ on $Y \times \mathbb{R}^{2} \times \hat{S} \times \hat{S}$ :

$$
\left(g,\left(y, w,\left(z_{1}, z_{2}\right)\right)\right) \mapsto\left(y, w,\left(s_{1} z_{1}, s_{2} z_{2}\right)\right),
$$

where $g=\left(s_{1}, s_{2}\right) \in T^{2}, y \in Y, w \in \mathbb{R}^{2}$, and $\left(z_{1}, z_{2}\right) \in \hat{S} \times \hat{S}$. It follows from our discussion above that $Y \times \mathbb{R}^{2} \times \hat{S} \times \hat{S}$ is real-analytic diffeomorphic to $Y \times Z=X$. Hence the proof is complete.

Corollary 9 Let $X$ be a Banach space of the form $X=Y \times Z$, where $Z$ is a separable infinite-dimensional space which is isomorphic to its cartesian square. Then, for each integer $n \geq 2$ there exists a real-analytic diffeomorphism $f: X \longrightarrow X$ of pure period $n$ such that $f$ has no fixed points.

Proof From the preceding theorem we know that there is a real-analytic free action $\Phi$ of the unit circle $T$ on the space $X$. Then it is clear that $x \mapsto \Phi\left(e^{2 \pi i / n}, x\right)$ is a real-analytic self-diffeomorphism of pure period $n$ and without fixed points.

Acknowledgements The authors wish to thank C. Bessaga for several valuable suggestions.

\section{References}

[1] D. Azagra, Smooth negligibility and subdifferential calculus in Banach spaces, with applications. Doctoral dissertation, Universidad Complutense de Madrid, December 1997.

[2] Diffeomorphisms between spheres and hyperplanes in infinite-dimensional Banach spaces. Studia Math. (2) 125(1997), 179-186.

[3] D. Azagra and T. Dobrowolski, Smooth negligibility of compact sets in infinite-dimensional Banach spaces, with applications. Math. Ann. (3) 312(1998), 445-463. 
[4] D. Azagra, J. Gómez, and J. A. Jaramillo, Rolle's theorem and negligibility of points in infinite-dimensional Banach spaces. J. Math. Anal. Appl. (2) 213(1997), 487-495.

[5] C. Bessaga, Every infinite-dimensional Hilbert space is diffeomorphic with its unit sphere. Bull. Acad. Polon. Sci. Sér. Sci. Math. Astronom. Phys. 14(1966), 27-31.

[6] Negligible sets in linear topological spaces. Bull. Acad. Polon. Sci. Sér. Sci. Math. Astronom. Phys. 16(1968), 117-119.

[7] Interplay Between Infinite-Dimensional Topology and Functional Analysis. Mappings $\overrightarrow{D e f i n e d}$ by Explicit Formulas and Their Applications. Topology Proc. 19(1994), 15-35.

[8] C. Bessaga and V. L. Klee, Two topological properties of topological linear spaces. Israel J. Math. 2(1964), 211-220

[9] Every non-normable Fréchet space is homeomorphic with all of its closed convex bodies. Math. Ann. 163(1966), 161-166.

[10] C. Bessaga and A. Pełczyński, Selected topics in infinite-dimensional topology. Monografie Matematyczne 58, Polish Scientific Publishers, Warsaw, 1975.

[11] D. Burghelea and N. H. Kuiper, Hilbert manifolds. Ann. of Math. 90(1969), 379-417.

[12] H. H. Corson and V. L. Klee, Topological classification of convex sets. Proc. Symp. Pure Math. 7, Amer. Math. Soc., Providence, RI, 1963, 37-51.

[13] R. Deville, G. Godefroy, and V. Zizler, Smoothness and renormings in Banach spaces. Pitman Monographies and Surveys in Pure and Applied Mathematics 64, 1993.

[14] J. Diestel, Geometry of Banach Spaces_Selected Topics. Lecture Notes in Math. 485, Springer-Verlag, New York, 1975.

[15] J. Dieudonné, Foundations of Modern Analysis. Academic Press, New York-London, 1960.

[16] T. Dobrowolski, Smooth and R-analytic negligibility of subsets and extension of homeomorphism in Banach spaces. Studia Math. 65(1979), 115-139.

[17] — Every Infinite-Dimensional Hilbert Space is Real-Analytically Isomorphic with Its Unit Sphere. J. Funct. Anal. 134(1995), 350-362.

[18] Relative Classification of Smooth Convex Bodies. Bull. Acad. Polon. Sci. Sér. Sci. Math. Astronom. Phys. 25(1977), 309-312.

[19] B. M. Garay, Cross sections of solution funnels in Banach spaces. Studia Math. 97(1990), $13-26$.

[20] Deleting Homeomorphisms and the Failure of Peano's Existence Theorem in Infinite-Dimensional Banach Spaces. Funkcialaj Ekvacioj 34(1991), 85-93.

[21] Parallelizability in Banach spaces I-II-III, Acta Math. Hungar., Proc Roy. Soc. Edinburgh, Proc. Banach Center Sem.

[22] K. Goebel and J. Wośko, Making a hole in the space. Proc. Amer. Math. Soc. 114(1992), 475-476.

[23] R. C. James, Weakly compact sets. Trans. Amer. Math. Soc. 113(1964), 129-140.

[24] K. John, H. Toruńczyck and V. Zizler, Uniformly smooth partitions of unity on superreflexive spaces. Studia Math. 70(1981), 129-137.

[25] V. L. Klee, Convex bodies and periodic homeomorphisms in Hilbert space. Trans. Amer. Math. Soc. 74(1953), 10-43.

[26] P. A. Smith, Fixed-point theorems for periodic transformations. Amer. J. Math. 63(1941), 1-8.

[27] J. J. Stoker, Unbounded convex point sets. Amer. J. Math. 62(1940), 165-179.

[28] E. F. Whittlesey, Analytic functions in Banach spaces. Proc. Amer. Math. Soc. 16(1965), 1077-1083.

Departamento de Análisis Matemático

Facultad de Ciencias Matemáticas

Universidad Complutense

Madrid 28040

Spain

email:daniel@sunam1.mat.ucm.es
Department of Mathematics

Pittsburg State University

Pittsburg, Kansas 66762

USA

email: tdobrowo@mail.pittstate.edu 